\title{
Effects of Mini-Basketball Training Program on Social Communication Impairment and Executive Control Network in Preschool Children with Autism Spectrum Disorder
}

\author{
Sixin Yang ${ }^{1,2}{ }^{\circ}$, Zhimei Liu ${ }^{1,2}$, Xuan Xiong ${ }^{1,2}$, Kelong Cai ${ }^{1,2}$, Lina Zhu ${ }^{3} \oplus$, Xiaoxiao Dong ${ }^{1,2}$, Jingui Wang ${ }^{1,2}$, \\ Hao Zhu ${ }^{1,2}$, Yifan Shi ${ }^{1,2}$ (D) and Aiguo Chen ${ }^{1,2,4, *(D)}$ \\ 1 College of Physical Education, Yangzhou University, Yangzhou 225127, China; \\ MX120200467@yzu.edu.cn (S.Y.); MX120170354@yzu.edu.cn (Z.L.); movmu7@sina.com (X.X.); \\ MX120170353@yzu.edu.cn (K.C.); DX120190065@yzu.edu.cn (X.D.); MX120170362@yzu.edu.cn (J.W.); \\ MX120180365@yzu.edu.cn (H.Z.); MX120190380@yzu.edu.cn (Y.S.) \\ 2 Institute of Sports, Exercise and Brain, Yangzhou University, Yangzhou 225127, China \\ 3 School of Physical Education and Sports Science, Beijing Normal University, Beijing 100000, China; \\ zhulina827@mail.bnu.edu.cn \\ 4 Chinese-Polish Laboratory of Sport and Brain Science, Yangzhou University, Yangzhou 225127, China \\ * Correspondence: agchen@yzu.edu.cn; Tel.: +86-1395-272-5968
}

Citation: Yang, S.; Liu, Z.; Xiong, X.; Cai, K.; Zhu, L.; Dong, X.; Wang, J.; Zhu, H.; Shi, Y.; Chen, A. Effects of Mini-Basketball Training Program on Social Communication Impairment and Executive Control Network in Preschool Children with Autism Spectrum Disorder. Int. J. Environ Res. Public Health 2021, 18, 5132. https://doi.org/10.3390/ ijerph18105132

Academic Editor: Maria Esposito

Received: 1 April 2021

Accepted: 5 May 2021

Published: 12 May 2021

Publisher's Note: MDPI stays neutral with regard to jurisdictional claims in published maps and institutional affiliations.

Copyright: (c) 2021 by the authors. Licensee MDPI, Basel, Switzerland. This article is an open access article distributed under the terms and conditions of the Creative Commons Attribution (CC BY) license (https:// creativecommons.org/licenses/by/ $4.0 /)$.
Abstract: This study evaluated the effect of a 12-week mini-basketball training program (MBTP) on social communication (SC) and the executive control network (ECN) in preschool children with autism spectrum disorders (ASD). We finally assigned 30 preschool children with ASD to an experiment group ( $n=15,12$ males, 3 females) or a control group ( $n=15,13$ males, 2 females). The experiment group participated in a 12-week MBTP (40-min sessions per day, 5 days a week), while the control group only received the institutional routine behavioral rehabilitation intervention. The SC of preschool children with ASD was measured using the Social Responsiveness Scale, Second Edition (SRS-2), whereas functional connectivity (FC) of the ECN was assessed using resting-state functional magnetic resonance imaging (rs-fMRI) at pre-and post-test. Our results showed that SC exhibited significant improvement in the intervention group, especially in SRS-2 total score, social cognition, and social communication. We found significantly enhanced functional connectivity between the right cerebellum and left inferior frontal gyrus in the experimental group, while functional connectivity between the left middle temporal gyrus and right cerebellum were decreased in the control group. Furthermore, there were no significant correlations between the change in SC scores and FC of the ECN. Altogether, this study provides valuable insights that a 12-week MBTP improves SC and functional connectivity of the ECN in preschool children with ASD. We further inferred that neural mechanisms might be associated with changing the ECN of preschool ASD children caused by the 12-week MBTP.

Keywords: mini-basketball; autism spectrum disorder; social communication; executive control network; functional connectivity

\section{Introduction}

Autism spectrum disorder (ASD) is a neurodevelopmental disorder of unknown etiology [1]. According to the latest statistics from the U.S. Centers for Disease Control and Prevention (CDC), the prevalence of ASD has increased to 1 in 54 children [2]. The rapidly growing incidence of this disease has aroused public concern. Social communication (SC) impairment is one of the main characteristics of ASD, and manifests as deficits in social cognition, pragmatics, language processing, and verbal and nonverbal communication [3]. Overwhelming evidence shows that SC deficit limits the individual development of ASD children, seriously affecting the quality of their family life [4], as well as posing a substantial economic burden on society [5]. Recent research suggests that early interventions in 
children diagnosed with ASD before age 3 can significantly improve cognitive, social, and adaptive functioning in children with ASD [6]; therefore, early intervention and treatment in preschool is desperately needed.

Recently, physical exercise programs have been widely used in the therapy of children with ASD, and more importantly, physical exercise has a significant effect on improving SC impairment [7-13]. Among them, mini-basketball has the inherent characteristics of basketball and is designed for children under 12 years old, which is parallel with the physical and mental developmental characteristics of children. Mounting evidence has demonstrated that a mini-basketball training program (MBTP) can effectively improve the core symptoms of children with ASD, as well as promote the development of physical fitness [14], cognitive function [15,16], and other aspects. Therefore, mini-basketball has been regarded as a promising and valuable means of intervention; however, the neural mechanism by which an MBTP improves SC impairment remains unclear.

The existing evidence strongly corroborates that ASD is associated with the functional brain network, especially in the executive control network (ECN). Studies have suggested that abnormalities in the ECN may be interpreted as a neural mechanism for SC impairment in ASD. Abnormal ECN functional connectivity in children with ASD has been associated with social behavior [17]. Based on neural connectivity, evidence from a categoricaldimensional hybrid model of ASD indicated that children with ASD showed linear relations between functional connectivity of the ECN and SRS score [18]. These findings suggest that changes in functional connectivity of the ECN contribute significantly to the development of SC in children with ASD. Furthermore, the plasticity of physical exercise on the functional connectivity of the ECN has been demonstrated [19-21]. Therefore, exploring the effect of an MBTP on the plasticity of the ECN is the first way to reveal the neuromechanics of physical exercise to improve the social communication impairment of preschool children with ASD.

Taken together, we aimed to explore the influence of the mini-basketball intervention on SC impairment and the ECN of preschool children with ASD, so as to reveal the physical exercise intervention and improve social communication impairment and the neural mechanism of preschool children with ASD, thus providing useful and new evidence. Herein, we hypothesize that a 12-week MBTP can improve the social communication impairment and ECN of preschool children with ASD. Further, improved social communication impairment in preschool children with ASD may be associated with changes in the ECN.

\section{Materials and Methods}

\subsection{Participants}

For this study, 94 preschoolers from two educational institutions were recruited. Finally, only 30 participants completed the study (Figure 1). Inclusion criteria were as follows: (1) Han Chinese; (2) children who meet DSM-V criteria and are diagnosed with moderate to severe ASD; (3) age 3-6 years; (4) guardians of all participants agreed subjects to join the study; and (5) meeting a scan criterion for MRI. Exclusion criteria comprised of (1) head trauma; (2) children with a history of neurological disease and/or psychotic disorders; (3) hearing and vision impairments; (4) taking any medication affecting the central nervous system in the past 6 months; (5) basketball training or taking part in physical exercise regularly in the past six months; and (6) no extremity disability. 


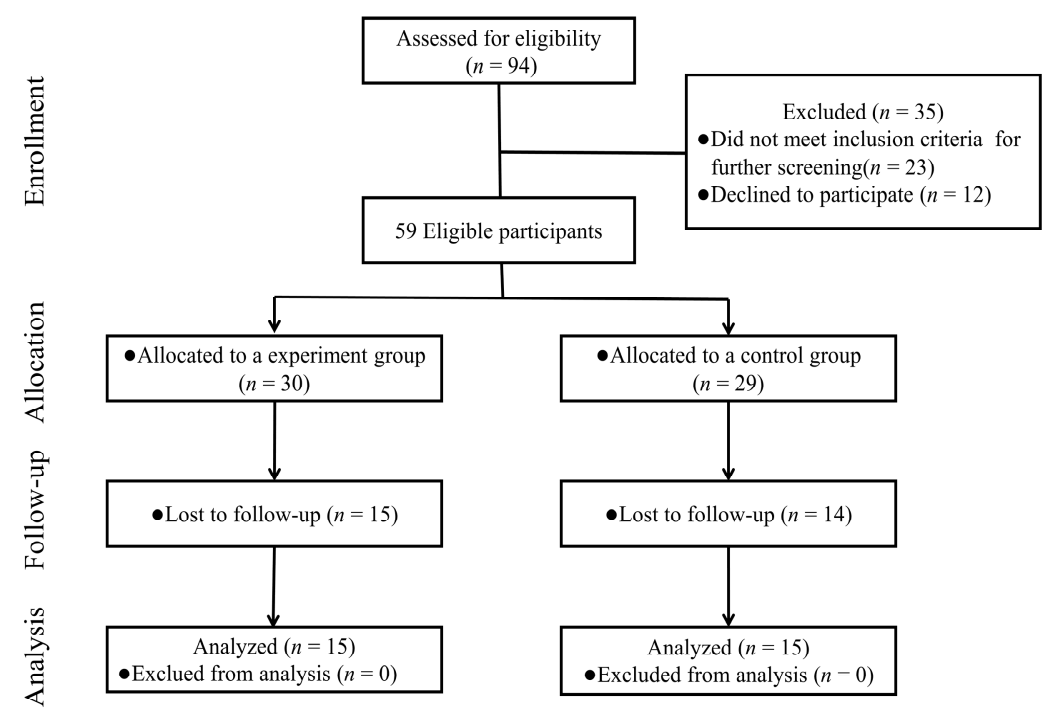

Figure 1. Participant follow chart.

The preschool students in two rehabilitation institutions were randomized into control and experimental groups. In particular, based on the rehabilitation of routine behavior in two groups, the experimental group participated a 12-week mini-basketball intervention, while the control group only received the institutional routine behavioral rehabilitation intervention. In total, 30 subjects were entered in this research, whereby the experimental group consisted of 15 participants (12 males and 3 females), whereas the control group also included 15 (13 males and 2 females). Reasons for screening were: (1) because the parents of the children with ASD did not complete the relevant questionnaires in the post-test, 27 subjects were not considered, and (2) T1-MPRAGE was missing for 2 subjects.

Previous studies have confirmed that the degree of disease [22], sleep impairment [23], eating behavior [24], and physical health and development of children with ASD [25] affected the core symptoms of ASD. Therefore, we herein selected the Childhood Autism Rating Scale (CARS) [26], the Children's Sleep Habits Questionnaire (CSHQ) [27], the Child Eating Behavior Questionnaire (CEBQ) [28], and the Children's Edition of National Physical Fitness Measurement Standard Manual (General Administration of Sport of China, 2003) to evaluate the corresponding indicators, aimed to balance the above confounding variables in the experimental grouping.

The CARS scale was filled out by the chief physician of the Child Autism Clinic in Yangzhou Maternal and Child Health Hospital following the evaluation of the clinical manifestations of children, and the evaluation doctors were consistent. The parents filled out the CSHQ and CEBQ scales according to the specific performance of subjects in daily life, and also the parents were consistent before and after filling in the scales.

\subsection{Study Design}

This study was conducted as a quasi-experimental trial that adopted a $2 \times 2$ mixed experimental design in which the factor "time" was within-subject factors, whereas the factor "group" was included as a between-subject factor. The protocol for this study was approved by the Ethics and Human Protection Committee of The Affiliated Hospital of Yangzhou University and registered in the Chinese Clinical Trial Registry (ChiCTR 1900024973). The parents or guardians of the study subjects signed the informed consent form before participation in the study.

\subsection{Mini-Basketball Training Program}

For this experiment, a mini-basketball training program was applied as a rehabilitation treatment to children with ASD as described previously [16,29-31]. The mini-basketball 
intervention program was mainly divided into three stages, in which each stage needed to have specific curriculum objectives; the details were as follows. (a) Stage 1 lasted for 2 weeks: to stimulate the interest of children in mini-basketball, standardize the classroom activities of children and parents (e.g., taking turns, waiting, and obeying.), and make it interesting and simple; (b) Stage 2 lasted for 8 weeks: to improve children's mini-basketball skills (e.g., dribbling, passing, shooting, etc.) and social communication skills (includes passing, catching and relay competitions between peers); (c) Stage 3 lasted for 2 weeks: to improve the ability of children's cooperation and collectivization, the content was based on mini-basketball group game (basketball dribbling relay, basketball passing relays, and basketball moving, shooting, etc.).

Each class of mini-basketball lasted for $40 \mathrm{~min}$, which consisted of four parts: "starting, warm-up, intervention, and relaxation." (a) Starting: children were asked to stand in line, accept roll call and conduct class greetings, etc.; (b) warm-up: this section mainly included jogging, stretching, the movement of four limbs, etc.; (c) intervention: simple basketball training in stage 1 , the mini-basketball skill learning in stage 2, the mini-basketball game in stage 3; (d) relaxation: finally, muscle relaxation and a class summary were carried out.

We set moderate exercise intensity as follows: $60-69 \%$ of maximum heart rate (MHR). The MHR was monitored using a POLAR M430 heart rate monitor and calculated with the formula: (MHR $=220$ - age of the participant) [32].

\subsection{Measurement of Social Communication}

The social communication ability of preschool children with ASD was assessed using the second version of the Social Response Scale (SRS-2) [33], which is a parent or teacher rating scale developed by John N. Constantino and Christian P. Gruber in 2012 to investigate the social ability of autistic children between 2 years and 6 months to adulthood. It consisted of a total of 65 questions using a 5-point Likert scale, with higher scores indicating more severe social impairment. The SRS-2 scale was filled out by parents according to the specific performance of the subjects in daily life. Furthermore, the parents were consistent before and after filling in the scales.

\section{5. rs-fMRI Data Collection and Processing}

During the data and image acquisition process, the MRI scanner ran loudly and took a long time, so it was impossible to ensure that every subject completed the scan successfully. Therefore, to increase the success rate of the scan, this experiment moderately deprived all subjects of sleep, sedated them, and made clear specific requirements to the guardians of the children with ASD the day before the MRI scan so that the subjects could go to bed late and get up early. Each subject received an enema of $10 \%$ chloral hydrate at an interval of 6 to $8 \mathrm{~h}, 0.3 \mathrm{~mL} / \mathrm{kg}(30 \mathrm{mg} / \mathrm{kg}$ ), with a maximum dose not exceeding $10 \mathrm{~mL}$. The nurse tested the level of consciousness of subjects after they fell asleep. If a mildly painful stimulus did not produce a conscious response, the subject was placed on his or her back in the MRI scanner by their guardian. Image data acquisition was performed in the MRI scanning room of the Affiliated Hospital of Yangzhou University using a 3.0T MRI scanner (GE Discovery MR750W 3.0T, Chicago, IL, USA). The data acquisition parameters for the structural MRI T1 scan were as follows: TR $/ \mathrm{TE}=7.2 / 3.1 \mathrm{~ms}$, matrix size $=256 \times 256,170$ interleaved slices, voxel size $1 \mathrm{~mm} \times 1 \mathrm{~mm} \times 3 \mathrm{~mm}$, and FOV $=256 \times 256 \mathrm{~mm}^{2}$ with 170 interleaved slices. The resting-state scan parameters included TR $/ \mathrm{TE}=2000 / 30 \mathrm{~ms}$, matrix size $=64 \times 64$, voxel size $=3.5 \mathrm{~mm} \times 3.5 \mathrm{~mm} \times 4 \mathrm{~mm}$, and FOV $=224 \times 224 \mathrm{~mm}^{2}$ with 28 slices.

The ROI was selected from the executive control brain network template (https: / / findlab.stanford.edu/functional_ROIs.html (accessed on: 1 October 2020)) of the center for functional imaging of neuropsychiatric diseases (Table 1). 
Table 1. Twelve ROIs of executive control network.

\begin{tabular}{cccc}
\hline ROI & Brain Hemispheres & Primary Regions & Brodmann' Area \\
\hline RECN1 & Right & orbitofrontal gyrus/dorsolateral & prefrontal cortex \\
RECN2 & Right & frontal pole/inferior frontal gyrus & $10 / 46 / 47$ \\
RECN3 & Right & inferior parietal lobule & $7 / 39 / 40$ \\
RECN4 & Right & middle temporal gyrus & $20 / 37$ \\
RECN5 & Left & cerebellum & - \\
RECN6 & Right & caudate nucleus & - \\
LECN1 & Left & orbitofrontal gyrus / dorsolateral & $8 / 9 / 46$ \\
LECN2 & Left & prefrontal cortex & $10 / 45 / 47$ \\
LECN3 & frontal pole/inferior frontal gyrus & $7 / 39 / 40$ \\
LECN4 & Left & inferior parietal lobule & $20 / 37$ \\
LECN5 & Right & middle temporal gyrus & - \\
LECN6 & Left & cerebellum & - \\
\hline
\end{tabular}

\subsection{Procedure}

The whole experiment consisted of three parts: pre-test, mini-basketball intervention, and post-test (Figure 1).

Pre-test: This part was performed one week before the formal experiment. We communicated entirely with the parents of the subjects and introduced the mini-basketball intervention to all children with ASD and their parents. At the same time, the demographic information of the subjects was investigated in detail, and their parents and evaluation doctors filled out the relevant questionnaires.

Mini-basketball intervention: In the experimental group, preschooler children with ASD were given a moderate-intensity mini-basketball training program for $40 \mathrm{~min}$ a day, 5 days a week, for 12 weeks, while the control group received rehabilitation of routine behavior.

Post-test: After completing the 12-week mini-basketball intervention, we then collected brain image data from preschool children with ASD. Finally, parents filled out the SRS-2 scale, and the parents who filled out the scale were the same person as the pre-test.

\subsection{Statistical Analysis}

First, a homogeneity test was performed on the demography data (age, sex, and BMI), as well as the CARS, physical assessment, CSHQ scores, and CEBQ scores of the experimental and control groups using an independent sample t-test with SPSS statistical software, version 22.0 (IBM, Armonk, NY, USA). The results are expressed as mean \pm standard deviation $(\mathrm{M} \pm \mathrm{SD}$ ). Second, repeated measures analysis of variance (ANOVA) was used to analyze the social communication impairment data, whereas effect sizes are presented as partial eta-squared (partial $\eta^{2}$ ). When a significant interaction was found, a simple effects analysis was conducted.

We employed the DPABI (http://rfmri.org/dpabi (accessed on: 1 October 2020)) software to calculate the functional connectivity of ECN, while a two-sample t-test based on GRETNA (http:/ / www.nitrc.org/projects/gretna/ (accessed on: 1 October 2020)) was used to test whether the functional connectivity between the 12 pairs of ROIs examined in the two groups of preschool children with ASD were homogeneous in pre-test. We also performed repeated measurement ANOVA on the functional connectivity between the 12 ROIs measured before and after in the two groups of preschool children with ASD using MATLAB software (MathWorks, Natick, MA, USA). Lastly, the brain regions with significant differences were obtained, and subsequently, their functional connectivity values were extracted to explore the changes in their intensity.

Finally, correlation analysis was used to examine the difference between the FC value of the ECN and the difference between the social communication performance, before and after 12-week MBTP. 


\section{Results}

\subsection{Participant Characteristics}

The demographic characteristics of the participants are summarized in Table 1. We found that participants exhibited no difference at baseline in terms of gender (Chi-square: $\left.\chi^{2}=0.24, p=0.62\right)$, age $(\mathrm{t}(28)=1.60, p=0.12>0.05)$, BMI $(\mathrm{t}(28)=-0.25, p=0.81>0.05)$, speed-agility $(\mathrm{t}(28)=1.22, p=0.23>0.05)$, muscular strength $(\mathrm{t}(28)=1.45, p=0.16>0.05)$, balance ability ( $\mathrm{t}(28)=0.21, p=0.84>0.05)$, CARS ( $\mathrm{t}(28)=-0.94, p=0.36>0.05)$, CSHQ $(\mathrm{t}(28)=-0.62, p=0.54>0.05)$, and CEBQ $(\mathrm{t}(28)=0.14, p=0.89>0.05)$ during the study period. Mean and standard deviation of physical fitness performance and the SRS-2 scores of all children are depicted in Table 2.

Table 2. Participant demographics evaluation results $(M \pm S D)$.

\begin{tabular}{ccc}
\hline & Control Group & Experiment Group \\
\hline$N$ & 15 & 15 \\
Gender(male/female) & $12 / 3$ & $13 / 2$ \\
Age & $5.03 \pm 0.55$ & $4.67 \pm 0.70$ \\
BMI & $15.75 \pm 0.67$ & $15.88 \pm 1.80$ \\
CARS $^{\text {a }}$ & $38.13 \pm 4.47$ & $39.80 \pm 5.24$ \\
CSHQ $^{\text {b }}$ & $56.47 \pm 5.04$ & $58.60 \pm 12.29$ \\
CEBQ $^{c}$ & $55.20 \pm 8.25$ & $54.40 \pm 20.05$ \\
20-m shuttle run (s) & $13.31 \pm 4.16$ & $11.83 \pm 2.23$ \\
Standing long jump (cm) & $50.23 \pm 28.75$ & $37.00 \pm 21.86$ \\
Sit-and-reach (cm) & $17.55 \pm 6.09$ & $19.61 \pm 2.43$ \\
Balance beam (s) & $21.92 \pm 36.00$ & $19.90 \pm 11.10$ \\
\hline
\end{tabular}

Note: a CARS: Childhood Autism Rating Scale; ${ }^{b}$ CSHQ: Children's Sleep Habits Questionnaire; ${ }^{c}$ CEBQ: Child Eating Behavior Questionnaire.

\subsection{Social Communication Performance}

Here, we adopted the repetitive measure analysis of variance method to explore the influence of the MBTP on the SRS-2 score of preschool children with ASD. The results of the interaction between groups and times can reveal whether the MBTP causes a change in the SRS-2 score. We found a significant group $\times$ time interaction on social communication total score $\left(\mathrm{F}(1,28)=7.77, p=0.009<0.01\right.$, partial $\left.\eta^{2}=0.22\right)$, social cognition $\left(\mathrm{F}(1,28)=9.97, p=0.004<0.01\right.$, partial $\left.\eta^{2}=0.26\right)$, and social communication $(\mathrm{F}(1,28)$ $=8.40, p=0.007<0.01$, partial $\left.\eta^{2}=0.23\right)$. Analysis of simple effects according to values (Table 2) revealed that the pre-test scores in all SRS-2 dimensions of the experimental and control groups were homogeneous $(p>0.05)$. However, there were significant differences in the total score of SRS-2 $(p=0.03<0.05)$ and the score of social communication dimension $(p=0.02<0.05)$ between the pre-test and post-test in the control group. In comparison, the score of the post-test was higher than that of the pre-test in the control group. Similarly, there was a significant difference between the pre-test and post-test scores of the experimental group in the dimension of social cognition $(p=0.01<0.05)$. Comparatively, the post-test score was lower compared with that of the pre-test score in the experimental group (A higher score means worse symptoms) (Table 3).

Table 3. Analysis of two groups for social communication variables ( $\pm S D$ ).

\begin{tabular}{ccccc}
\hline & \multicolumn{2}{c}{ Experiment Group $(\boldsymbol{n = 1 5 )}$} & \multicolumn{2}{c}{ Control Group $(\boldsymbol{n}=\mathbf{1 5})$} \\
\cline { 2 - 5 } & Baseline & Post-Test & Baseline & Post-Test \\
\hline SRS-2 T-score & $89.70 \pm 25.89$ & $81.50 \pm 4.55$ & $85.30 \pm 20.04$ & $97.30 \pm 21.35$ \\
Social cognition & $19.50 \pm 5.59$ & $16.50 \pm 6.07$ & $17.30 \pm 3.56$ & $19.30 \pm 3.89$ \\
Social communication & $31.70 \pm 1.86$ & $28.40 \pm 9.66$ & $30.90 \pm 8.06$ & $35.70 \pm 8.09$ \\
Social motivation & $14.40 \pm 4.72$ & $12.30 \pm 3.84$ & $14.40 \pm 4.39$ & $14.50 \pm 4.19$ \\
Autistic mannerisms & $12.30 \pm 5.04$ & $13.20 \pm 5.62$ & $12.30 \pm 5.36$ & $16.40 \pm 6.15$ \\
Social awareness & $11.90 \pm 2.80$ & $11.20 \pm 3.61$ & $10.50 \pm 2.45$ & $11.40 \pm 2.59$ \\
\hline
\end{tabular}




\subsection{Executive Control Network}

There was no significant difference in 12-pair ROI-ROI for the ECN in preschool children with ASD at baseline. We used ANOVA statistical function in MATLAB to perform repeated measures analysis of variance of the functional connectivity matrix for the 12 regions of interest (ROI) in the ECN generated by each participant. We subsequently observed significant differences in functional connectivity of 2-pair ROI-ROI in the ECN: left inferior frontal gyrus and right cerebellum $(p=0.03<0.05)$, left middle temporal gyrus and right cerebellum $(p=0.01<0.05)$ (Table 4$)$.

Table 4. Function connectivity results for 12 ROI-ROI changes in ECN, segregated by time point.

\begin{tabular}{|c|c|c|c|c|c|c|c|c|c|c|c|c|}
\hline & RECN1 & RECN2 & RECN3 & RECN4 & RECN5 & RECN6 & LECN1 & LECN2 & LECN3 & LECN4 & LECN5 & LECN6 \\
\hline \multicolumn{13}{|l|}{ RECN1 } \\
\hline RECN2 & 0.67 & & & & & & & & & & & \\
\hline RECN3 & 0.51 & 0.19 & & & & & & & & & & \\
\hline RECN4 & 0.73 & 0.96 & 0.48 & & & & & & & & & \\
\hline RECN5 & 0.77 & 0.71 & 0.80 & 0.37 & & & & & & & & \\
\hline RECN6 & 0.76 & 0.22 & 1.00 & 0.61 & 0.90 & & & & & & & \\
\hline LECN1 & 0.45 & 0.90 & 0.63 & 0.24 & 0.61 & 0.87 & & & & & & \\
\hline LECN2 & 0.78 & 0.34 & 0.75 & 0.76 & 0.40 & 0.73 & 0.41 & & & & & \\
\hline LECN3 & 0.38 & 0.77 & 0.57 & 0.65 & 0.46 & 0.45 & 0.41 & 0.87 & & & & \\
\hline LECN4 & 0.48 & 0.91 & 0.08 & 0.81 & 0.67 & 0.66 & 0.24 & 0.25 & 0.20 & & & \\
\hline LECN5 & 0.17 & 0.06 & 0.30 & 0.16 & 0.78 & 0.76 & 0.50 & 0.03 * & 0.05 & 0.01 * & & \\
\hline LECN6 & 0.05 & 0.79 & 0.73 & 0.61 & 0.32 & 0.81 & 0.73 & 0.43 & 0.31 & 0.98 & 0.50 & \\
\hline
\end{tabular}

Note: “*” indicates $p<0.05$ (uncorrected).

The mean comparison of the functional connectivity between the two pairs of ROIs demonstrated that the functional connectivity between the left inferior frontal gyrus and right cerebellum of the ASD children in the experimental group were enhanced, while the functional connectivity between the left middle temporal gyrus and right cerebellum were decreased in the control group (Figure 2).
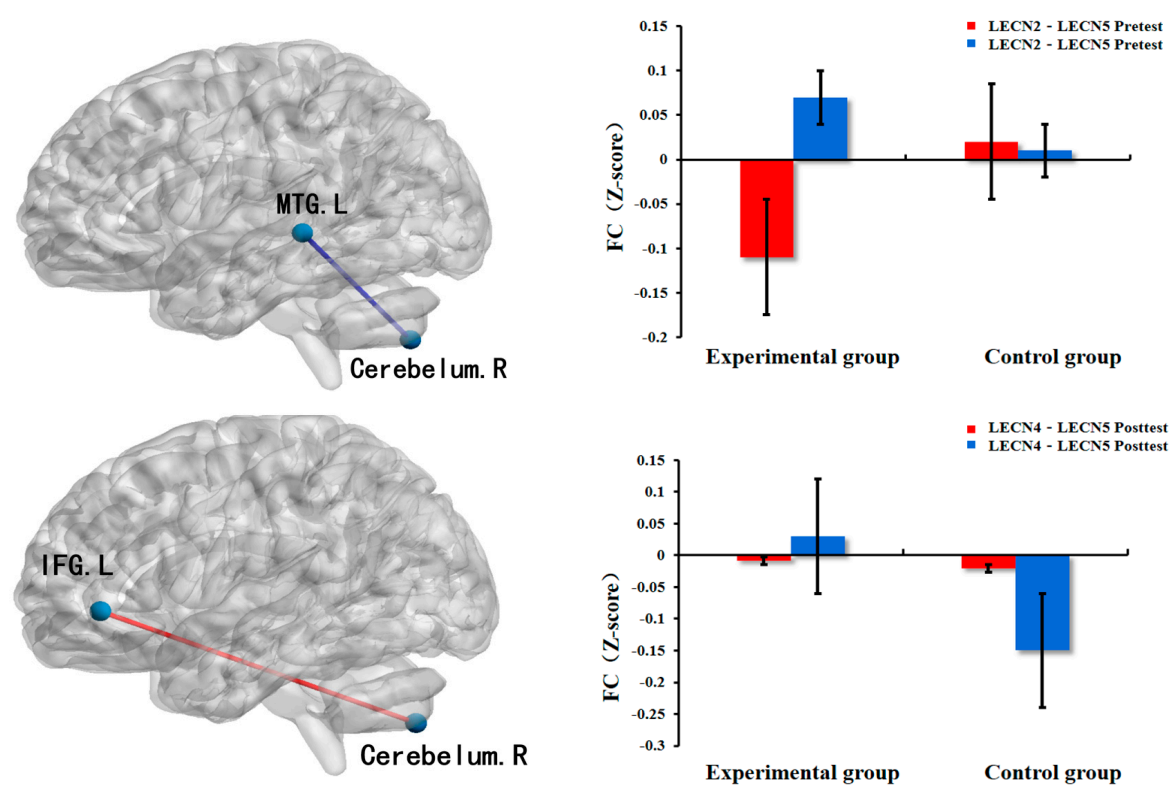

(a)

(b)

Figure 2. ECN changes caused by exercise intervention. Note: (a) left middle frontal gyrus, MFG.L, left middle temporal gyrus, MTG.L, right cerebellum, R, the blue nodes indicate the left ECN, the red line indicates FC enhancement, the blue line indicates FC decreased; (b) difference in FC values of ECN between the experimental group and the control group. 


\subsection{Correlation between Functional Connectivity of ECN and Behavioral Performance}

Correlation analysis was used to examine the difference between the FC value of $\mathrm{ECN}$ and the difference between the social communication performance, before and after 12-week MBTP. In order to avoid confusing the influence of variables, we took the height, weight, BMI, CARS score, CEBQ score, and CSHQ score as control variables for analysis. Our results showed the difference between the FC of the right cerebellum and left inferior frontal gyrus was not significantly correlated with the difference in the social cognition $(\mathrm{r}=-0.20, p=0.35)$, social communication $(\mathrm{r}=-0.14, p=0.52)$, total scores of SRS-2 $(\mathrm{r}=-0.16, p=0.46)$, the difference between the FC of right cerebellum and left middle temporal gyrus was not significantly correlated with the difference in the social cognition $(\mathrm{r}=-0.29, p=0.16)$, social communication $(\mathrm{r}=-0.32, p=0.13)$, total scores of SRS-2 $(\mathrm{r}=-0.25, p=0.24)$.

\section{Discussion}

This study was designed to explore the effects of 12-week MBTP on SC and functional connectivity of the ECN in children with ASD. In particular, one group, the experimental group, received a 12-week MBTP based on routine rehabilitation, while the other group, the control, received only conventional rehabilitation. In this work, we also tried to control the confounding variables as much as possible. Therefore, the findings of this study can reveal the neural basis of SC improvement due to the mini-basketball training program.

\subsection{Behavioral Performance}

In the present investigation, we found that 12-week MBTP improved the social communication impairment of preschool children with ASD. Previous studies have also elucidated that exercises such as mini-basketball [15], aquatic program [34], and Judo program [35] intervention can improve the social communication impairment of children with ASD. Thus, physical exercise is a promising method of rehabilitation for children with autism, and is worthy of further research.

Notably, the MBTP significantly improved the social cognition and social communication sub-dimensions of children with ASD. There are several plausible explanations for these observations. For example, research has shown that social cognition is the ability to recognize, manipulate, and respond to socially relevant information, thus creating complex performance characteristics [36]. Therefore, the improvement of the social cognitive ability of preschoolers with ASD may perhaps be due to the process of the MBTP because children need to identify the demonstration actions of teachers to imitate, which is also a process of social information processing [37]. Besides, a large number of social factors was contained in the process of the MBTP, such as children needing to accept roll call, conduct class greetings, pass and catch between peers, etc.; participation in this could provide opportunities to establish social relationships with their coach or peers, to enhance their social skills [38]. Recent studies tend to support this view, and hence children need to watch and cooperate with their peers in order to increase their social behavior such as in karate training [10]. Similar findings have been reported in water exercise swimming programs, containing a rich social communication environment [8].

We also identified no significant improvement in social motivation, autistic mannerisms, and social awareness. However, in one study, horseback riding intervention was found to markedly improve social motivation in children with ASD [11]. Thus, horseback riding was considered a rewarding stimulus, and children with ASD needed stronger motivation to participate, while the MBTP mainly focused on fine movement. This result may be ascribed to differences in the content of the intervention program. Additionally, autistic mannerisms and social awareness showed a trend of improvement although it was not statistically significant. We therefore assume that extending the period of the MBTP may cause a more significant effect, but more evidence is needed to verify this conjecture.

Interestingly, we have obtained some meaningful speculations. The content of the MBTP contained multiple elements of intervention; children with ASD were not only 
required to run, jump and other basic movements, but also to touch the ball and respond to instructions and other multifaceted stimulation exercises during this procedure; therefore, an MBTP may be beneficial to the development of the sensory and perceptual ability of children with ASD. The children need learn new movement and master the connection between movements in the process of the MBTP, which required a high level of cognition and control, and this may be beneficial for improving cognition function in children with ASD. In short, an MBTP may play a positive role in improving ASD-like behaviors. Collectively, based on the above findings, we uncovered that the MBTP played a positive role in improving social communication impairment in children with ASD, and considering factors such as program design and the content of the intervention cycle may also influence the intervention. Therefore, in future studies, there is a need to establish a more perfect intervention program to evaluate the efficacy of the improvement.

\subsection{Executive Control Network}

Evidence from exercise and brain plasticity shows that physical exercise can cause plasticity changes in the functional connectivity of the ECN [21,39]. Remarkably, our results support existing evidence that exercise intervention induces changes in the functional connectivity of the ECN. We found an increase in functional connectivity of the ECN between the right cerebellum and left inferior frontal gyrus. We subsequently inferred that this result might be attributed to the MBTP.

Multiple recent studies have shown that brain development in children with ASD has poor connectivity between regions; among them, there is evidence that the frontal and subcortical cerebellar regions exhibit inadequate connectivity [40,41]. Herein, we found enhanced functional connectivity in the right cerebellum and left inferior frontal gyrus in preschoolers with ASD after 12-week MBTP, suggesting that exercise intervention optimized the abnormalities of functional connectivity in ECN. Several studies have demonstrated that the cerebellum is a key brain region involved in motor function such as coordination and balance [42,43]. Further, the cerebellum is functionally connected to the frontal lobe [44], and evidence of co-activation of the cerebellum and the frontal lobe has also been found in functional neuroimaging $[45,46]$. Therefore, the enhanced cerebellar and frontal functional connectivity in preschoolers with ASD is the result of the 12-week MBTP.

In addition, studies have identified that children with ASD exhibit developmental disorders in the temporal lobe [47], and the functional connectivity between the temporal lobe and other brain regions decreases with age compared with normal children [48]. In this study, compared with the control group, the MBTP enhanced the functional connectivity between the left middle temporal gyrus and right cerebellum in the experimental group of preschoolers with ASD, thereby inhibiting the injury trend of functional connectivity deficiency. Similarly, previous studies have also confirmed that exercise can improve the abnormal functional connectivity between the temporal lobe brain region and related brain regions [19,49], which provides supportive evidence for the results of this study. Overall, this study found a positive effect of exercise on ECN in preschoolers with ASD, providing valuable and novel evidence for exercise and plasticity of the brain on children with ASD.

\subsection{Neural Mechanism of MBTP on Social Communication Impairment in Preschool Children with ASD}

Our finding revealed that there was no statistically significant correlation between the improvement of functional connectivity within the ECN and the improvement of SC, and it was inconsistent with previous studies. Several reasonable explanations that might answer this question exist. For instance, ECN-related brain regions, such as the cerebellum, frontal lobe and temporal lobe, are all involved in the formation of the "social brain" network [50-52], and many studies have also suggested that these brain regions are the neural basis of social communication impairment in ASD [18,53-58]. Elsewhere, functional connectivity in the right prefrontal, posterior, and inferior frontal gyrus was negatively correlated with social communication impairment scores. Moreover, longitudinal studies indicated that mini-basketball can promote social development by improving the structure 
of white matter [9]. Regrettably, the current study hoped to explore the neural basis of improving social communication impairment by mini-basketball from the perspective of functional brain network, but there was no statistically significant correlation between improved functional connectivity of the ECN and better performance of social communication impairment in our results. Fortunately, both showed a positive trend of improvement, which indicated that the neural mechanism of the MBTP on social interaction disorder of autistic preschool children may be the improvement of the ECN. We surmised that the reason for it not being statistically significant lies in small sample size or an imperfect MBTP. Thus, future studies will be further explored and refined, and reveal the functional brain network mechanism of the MBTP to improve social communication.

\subsection{Limition}

We believe that the limitations of this study is worth further discussion. First, although MBTP has a positive impact on the SC impairment of preschool children with ASD, it does not seem to be comprehensive (no significant statistically improvement in social motivation, social awareness sub-dimensions were shown). In the future research, we still need to think deeply and optimize our own program, hoping to play a more effective role in the rehabilitation of children with ASD. Second, it is a subjective measure that SRS-2 was administered by parents of the participating children, but we must admit that there are no other tools to help us evaluate ASD children more objectively.

\section{Conclusions}

In summary, this study demonstrates that social communication impairment was improved, and functional connectivity of the ECN was optimized after establishing an exercise regimen in preschool children with ASD. Notably, an MBTP is a valuable exercise intervention to improve social communication impairment, especially in children with ASD; this work provides an important baseline for future research in exploring the neural mechanisms by which exercise improves social communication impairment. Finally, our study has important implications for practice to the development and application of rehabilitation programs for children with ASD.

Author Contributions: Conceptualization, S.Y. and X.X.; methodology, S.Y. and K.C.; software, X.X.; validation, K.C., J.W. and Z.L.; formal analysis, S.Y., K.C. and J.W.; investigation, Z.L.; and Y.S.; resources, A.C. and X.X.; data curation, S.Y. and K.C.; writing-original draft preparation, S.Y.; writing review and editing, L.Z., H.Z. and A.C.; visualization, Y.S. and X.D.; supervision, X.X.; project administration, K.C.; funding acquisition, A.C. All authors have read and agreed to the published version of the manuscript.

Funding: This research was supported partly by grants received by Aiguo Chen from the National Natural Science Foundation of China (31771243) and the Fok Ying Tung Education Foundation (141113).

Institutional Review Board Statement: The study was conducted according to the guidelines of the Declaration of Helsinki, and approved by the ethics and human protection committees of the Affiliated Hospital of Yangzhou University (protocol code: 2017-YKL045-01 and date of approval: 7 January 2017).

Informed Consent Statement: Informed consent was obtained from all subjects involved in the study.

Data Availability Statement: The data presented in this study are available on request from the corresponding author.

Acknowledgments: The authors thank the children, guardians, staff, and investigators at the study sites for their contributions to this study.

Conflicts of Interest: The authors declare that the research was conducted in the absence of any commercial or financial relationships that could be construed as a potential conflict of interest. 


\section{References}

1. Blenner, S.; Reddy, A.; Augustyn, M. Diagnosis and management of autism in childhood. BMJ 2011, 343, d6238. [CrossRef]

2. Maenner, M.J.; Shaw, K.A.; Baio, J.; Washington, A.; Patrick, M.; DiRienzo, M.; Christensen, D.L.; Wiggins, L.D.; Pettygrove, S.; Andrews, J.G.; et al. Prevalence of Autism Spectrum Disorder among Children Aged 8 Years-Autism and Developmental Disabilities Monitoring Network, 11 Sites, United States, 2016. MMWR. Surveill. Summ. 2020, 69, 1-12. [CrossRef]

3. Landa, R.J.; Holman, K.C.; Garrett-Mayer, E. Social and Communication Development in Toddlers with Early and Later Diagnosis of Autism Spectrum Disorders. Arch. Gen. Psychiatry 2007, 64, 853-864. [CrossRef]

4. Padden, C.; James, J.E. Stress among Parents of Children with and without Autism Spectrum Disorder: A Comparison Involving Physiological Indicators and Parent Self-Reports. J. Dev. Phys. Disabil. 2017, 29, 567-586. [CrossRef]

5. Baxter, A.J.; Brugha, T.S.; Erskine, H.E.; Scheurer, R.W.; Vos, T.; Scott, J.G. The epidemiology and global burden of autism spectrum disorders. Psychol. Med. 2015, 45, 601-613. [CrossRef]

6. Ming, X.; Hashim, A.; Fleishman, S.; West, T.; Kang, N.; Chen, X.; Zimmerman-Bier, B. Access to specialty care in autism spectrum disorders-A pilot study of referral source. BMC Health Serv. Res. 2011, 11, 99. [CrossRef]

7. Sotoodeh, M.S.; Arabameri, E.; Panahibakhsh, M.; Kheiroddin, F.; Mirdoozandeh, H.; Ghanizadeh, A. Effectiveness of yoga training program on the severity of autism. Complement. Ther. Clin. Pract. 2017, 28, 47-53. [CrossRef]

8. Pan, C.-Y. Effects of water exercise swimming program on aquatic skills and social behaviors in children with autism spectrum disorders. Autism 2010, 14, 9-28. [CrossRef]

9. Cai, K.; Yu, Q.; Herold, F.; Liu, Z.; Wang, J.; Zhu, L.; Xiong, X.; Chen, A.; Müller, P.; Kramer, A.F.; et al. Mini-Basketball Training Program Improves Social Communication and White Matter Integrity in Children with Autism. Brain Sci. 2020, 10, 803. [CrossRef]

10. Bahrami, F.; Movahedi, A.; Marandi, S.M.; Sorensen, C. The Effect of Karate Techniques Training on Communication Deficit of Children with Autism Spectrum Disorders. J. Autism Dev. Disord. 2016, 46, 978-986. [CrossRef]

11. Bass, M.M.; Duchowny, C.A.; Llabre, M.M. The Effect of Therapeutic Horseback Riding on Social Functioning in Children with Autism. J. Autism Dev. Disord. 2009, 39, 1261-1267. [CrossRef]

12. Ketcheson, L.; Hauck, J.L.; Ulrich, D. The levels of physical activity and motor skills in young children with and without autism spectrum disorder, aged 2-5 years. Autism 2018, 22, 414-423. [CrossRef]

13. Reinders, N.J.; Branco, A.; Wright, K.; Fletcher, P.C.; Bryden, P.J. Scoping Review: Physical Activity and Social Functioning in Young People with Autism Spectrum Disorder. Front. Psychol. 2019, 10, 120. [CrossRef]

14. Cai, K.-L.; Wang, J.-G.; Liu, Z.-M.; Zhu, L.-N.; Xiong, X.; Klich, S.; Maszczyk, A.; Chen, A.-G. Mini-Basketball Training Program Improves Physical Fitness and Social Communication in Preschool Children with Autism Spectrum Disorders. J. Hum. Kinet. 2020, 73, 267-278. [CrossRef]

15. Wang, J.-G.; Cai, K.-L.; Liu, Z.-M.; Herold, F.; Zou, L.; Zhu, L.-N.; Xiong, X.; Chen, A.-G. Effects of Mini-Basketball Training Program on Executive Functions and Core Symptoms among Preschool Children with Autism Spectrum Disorders. Brain Sci. 2020, 10, 263. [CrossRef]

16. Zhu, Y.; Xu, C.; Wan, Q.; Guo, L.Y.; Sean, X.C. Effects of Adapted Physical Exercise Intervention on Visual Working Memory in Children with Autism Spectrum Disorder. China Sport Sci. Technol. 2017, 53, 55-62. [CrossRef]

17. Ma, Z.H.; Cao, Q.J.; Yan, C.G.; Mei, T.; Lu, B.; Liu, J.R.; Yang, L.; Wang, H.; Tang, X.Z.; Ji, Y.Z.; et al. Functional connectivity characteristics of executive control network in patients with high-functioning autism aged 6-18 years. Chinese Ment. Health J. 2019, 33, 401-405. [CrossRef]

18. Elton, A.; Di Martino, A.; Hazlett, H.C.; Gao, W. Neural Connectivity Evidence for a Categorical-Dimensional Hybrid Model of Autism Spectrum Disorder. Biol. Psychiatry 2016, 80, 120-128. [CrossRef]

19. Chen, A.G.; Zhu, L.N.; Xiong, X.; Li, Y. Effects of Aerobic Exercise on Executive Control and its Brain Network in Deaf Children. Sports Sci. 2016, 37, 94-101. [CrossRef]

20. Liu, J.; Chen, L.; Tu, Y.; Chen, X.; Hu, K.; Tu, Y.; Lin, M.; Xie, G.; Chen, S.; Huang, J.; et al. Different exercise modalities relieve pain syndrome in patients with knee osteoarthritis and modulate the dorsolateral prefrontal cortex: A multiple mode MRI study. Brain, Behav. Immun. 2019, 82, 253-263. [CrossRef]

21. Chen, A.G.; Zhu, L.N.; Xiong, X.; Li, Y. Acute aerobic exercise alters executive control network in preadolescent children. Rev. Psicol. Deporte 2017, 26, 132-137.

22. Hodges, H.; Fealko, C.; Soares, N. Autism spectrum disorder: Definition, epidemiology, causes, and clinical evaluation. Transl. Pediatr. 2020, 9, S55-S65. [CrossRef] [PubMed]

23. Veatch, O.J.; Sutcliffe, J.S.; Warren, Z.E.; Keenan, B.T.; Potter, M.H.; Malow, B.A. Shorter sleep duration is associated with social impairment and comorbidities in ASD. Autism Res. 2017, 10, 1221-1238. [CrossRef] [PubMed]

24. Marshall, J.V.; Ware, R.S.; Ziviani, J.M.; Hill, R.J.; Dodrill, P.M.M. Efficacy of interventions to improve feeding difficulties in children with autism spectrum disorders: A systematic review and meta-analysis. Child Care Heath Dev. 2015, 41, 278-302. [CrossRef]

25. Tyler, K.; Macdonald, M.; Menear, K. Physical Activity and Physical Fitness of School-Aged Children and Youth with Autism Spectrum Disorders. Autism Res. Treat. 2014, 2014, 312163. [CrossRef]

26. Schopler, E.; Reichler, R.J.; DeVellis, R.F.; Daly, K. Toward objective classification of childhood autism: Childhood Autism Rating Scale (CARS). J. Autism Dev. Disord. 1980, 10, 91-103. [CrossRef] 
27. Owens, J.A.; Spirito, A.; McGuinn, M. The Children's Sleep Habits Questionnaire (CSHQ): Psychometric properties of a survey instrument for school-aged children. Sleep 2000, 23, 1043-1051. [CrossRef]

28. Wardle, J.; Guthrie, C.A.; Sanderson, S.; Rapoport, L. Development of the Children's Eating Behaviour Questionnaire. J. Child Psychol. Psychiatry 2001, 42, 963-970. [CrossRef]

29. Lambert, J.M.; Copeland, B.A.; Karp, E.L.; Finley, C.I.; Houchins-Juarez, N.J.; Ledford, J.R. Chaining Functional Basketball Sequences (with Embedded Conditional Discriminations) in an Adolescent with Autism. Behav. Anal. Pr. 2016, 9, 199-210. [CrossRef]

30. Fotrousi, F.; Bagherly, J.; Ghasemi, A. The Compensatory Impact of Mini-Basketball Skills on the Progress of Fundamental Movements in Children. Procedia-Soc. Behav. Sci. 2012, 46, 5206-5210. [CrossRef]

31. Zhang, Z.Y.; Deng, S.H. Case Study on Sports Games Intervention of Autistic Children. China Sport Sci. Technol. 2010, 30, 386-390. [CrossRef]

32. Ferreira, J.P.; Ghiarone, T.; Júnior, C.R.C.; Furtado, G.E.; Carvalho, H.M.; Machado-Rodrigues, A.M.; Toscano, C.V.A. Effects of Physical Exercise on the Stereotyped Behavior of Children with Autism Spectrum Disorders. Medicina 2019, 55, 685. [CrossRef]

33. Constantino, J.; Gruber, C.P. The Social Responsiveness Scale (SRS) Manual; Western Psychological Services: Los Angeles, CA, USA, 2005.

34. Pan, C.-Y. The efficacy of an aquatic program on physical fitness and aquatic skills in children with and without autism spectrum disorders. Res. Autism Spectr. Disord. 2011, 5, 657-665. [CrossRef]

35. Rivera, P.; Renziehausen, J.; Garcia, J.M. Effects of an 8-Week Judo Program on Behaviors in Children with Autism Spectrum Disorder: A Mixed-Methods Approach. Child Psychiatry Hum. Dev. 2020, 51, 734-741. [CrossRef]

36. Adolphs, R. The neurobiology of social cognition. Curr. Opin. Neurobiol. 2001, 11, 231-239. [CrossRef]

37. Ferraioli, S.J.; Harris, S.L. Treatments to Increase Social Awareness and Social Skills; Springer: Boston, MA, USA, 2010; pp. 171-196.

38. Taub, D.E.; Greer, K.R. Physical activity as a normalizing experience for school-age children: With physical disa-bilities: Implications for legitimation of social identity and enhancement for social ties. J. Sport Soc. Issues 2000. [CrossRef]

39. Prehn, K.; Lesemann, A.; Krey, G.; Witte, A.V.; Köbe, T.; Grittner, U.; Flöel, A. Using resting-state fMRI to assess the effect of aerobic exercise on functional connectivity of the DLPFC in older overweight adults. Brain Cogn. 2019, 131, 34-44. [CrossRef]

40. Geschwind, D.H.; Levitt, P. Autism spectrum disorders: Developmental disconnection syndromes. Curr. Opin. Neurobiol. 2007, 17, 103-111. [CrossRef]

41. Courchesne, E.; Pierce, K. Why the frontal cortex in autism might be talking only to itself: Local over-connectivity but long-distance disconnection. Curr. Opin. Neurobiol. 2005, 15, 225-230. [CrossRef]

42. Fatemi, S.H.; Aldinger, K.A.; Ashwood, P.; Bauman, M.L.; Blaha, C.D.; Blatt, G.J.; Chauhan, A.; Chauhan, V.; Dager, S.R.; Dickson, P.E.; et al. Consensus Paper: Pathological Role of the Cerebellum in Autism. Cerebellum 2012, 11, 777-807. [CrossRef]

43. Manto, M.; Bower, J.M.; Conforto, A.B.; Delgado-García, J.M.; Da Guarda, S.N.F.; Gerwig, M.; Habas, C.; Hagura, N.; Ivry, R.B.; Mariën, P.; et al. Consensus Paper: Roles of the Cerebellum in Motor Control-The Diversity of Ideas on Cerebellar Involvement in Movement. Cerebellum 2012, 11, 457-487. [CrossRef] [PubMed]

44. Diamond, A. Close Interrelation of Motor Development and Cognitive Development and of the Cerebellum and Prefrontal Cortex. Child Dev. 2000, 71, 44-56. [CrossRef]

45. Schlosser, R.; Hutchinson, M.; Joseffer, S.; Rusinek, H.; Saarimaki, A.; Stevenson, J.; Dewey, S.L.; Brodie, J.D. Functional magnetic resonance imaging of human brain activity in a verbal fluency task. J. Neurol. Neurosurg. Psychiatry 1998, 64, 492-498. [CrossRef]

46. Raichle, M.E.; Fiez, J.A.; Videen, T.O.; MacLeod, A.-M.K.; Pardo, J.V.; Fox, P.T.; Petersen, S.E. Practice-related Changes in Human Brain Functional Anatomy during Nonmotor Learning. Cereb. Cortex 1994, 4, 8-26. [CrossRef]

47. Hetzler, B.E.; Griffin, J.L. Infantile autism and the temporal lobe of the brain. J. Autism Dev. Disord. 1981, 11, 317-330. [CrossRef]

48. Xu, J.; Wang, C.; Xu, Z.; Li, T.; Chen, F.; Chen, K.; Gao, J.; Wang, J.; Hu, Q. Specific Functional Connectivity Patterns of Middle Temporal Gyrus Subregions in Children and Adults with Autism Spectrum Disorder. Autism Res. 2019, 13, 410-422. [CrossRef]

49. Eli, R.; Ezhu, X.; Eyin, S.; Eniu, Y.; Ezheng, Z.; Ehuang, X.; Ewang, B.; Eli, J. Multimodal intervention in older adults improves resting-state functional connectivity between the medial prefrontal cortex and medial temporal lobe. Front. Aging Neurosci. 2014, 6, 39. [CrossRef]

50. Blakemore, S.-J. The social brain in adolescence. Nat. Rev. Neurosci. 2008, 9, 267-277. [CrossRef]

51. Adolphs, R. Cognitive neuroscience of human social behaviour. Nat. Rev. Neurosci. 2003, 4, 165-178. [CrossRef]

52. Brothers, L.; Ring, B.; Kling, A. Response of neurons in the macaque amygdala to complex social stimuli. Behav. Brain Res. 1990, 41, 199-213. [CrossRef]

53. Pelphrey, K.A.; Morris, J.P.; McCarthy, G. Neural basis of eye gaze processing deficits in autism. Brain 2005, 128, 1038-1048. [CrossRef] [PubMed]

54. Williams, J.H.; Waiter, G.D.; Gilchrist, A.; Perrett, D.I.; Murray, A.D.; Whiten, A. Neural mechanisms of imitation and 'mirror neuron' functioning in autistic spectrum disorder. Neuropsychologia 2006, 44, 610-621. [CrossRef] [PubMed]

55. Pierce, K.; Haist, F.; Sedaghat, F.; Courchesne, E. The brain response to personally familiar faces in autism: Findings of fusiform activity and beyond. Brain 2004, 127, 2703-2716. [CrossRef] [PubMed]

56. Wang, A.T.; Lee, S.S.; Sigman, M.; Dapretto, M. Reading Affect in the Face and Voice. Arch. Gen. Psychiatry 2007, 64, 698-708. [CrossRef] 
57. Whitney, C.; Jefferies, E.; Kircher, T. Heterogeneity of the Left Temporal Lobe in Semantic Representation and Control: Priming Multiple versus Single Meanings of Ambiguous Words. Cereb. Cortex 2010, 21, 831-844. [CrossRef]

58. Levisohn, L.; Cronin-Golomb, A.; Schmahmann, J.D. Neuropsychological consequences of cerebellar tumour resection in children. Brain 2000, 123, 1041-1050. [CrossRef] 